\title{
Study on Tool Path Optimization in Multi-axis NC Machining
}

\author{
Xinghua Niu ${ }^{1,2, a}$, Tianding Wang ${ }^{1,2}$, Bofei Shen ${ }^{1,2}$, Tiemin Zhao ${ }^{1,2}$ and Qianyou Huang ${ }^{1,2}$ \\ ${ }^{1}$ Tianjin Key Laboratory of the Design and Intelligent Control of the Advanced Mechatronical System, Tianjin 300384, China \\ ${ }^{2}$ Tianjin University of Technology, Tianjin 300384, China
}

\begin{abstract}
This paper presents a new generation algorithm for tool path based on the optimization of traditional algorithms. Then, the tool path on an impeller is generated with UG software, and it is used to make contrasts and verifications for the effect of optimization. Finally, VERICUT software with the function of the simulating on the whole manufacturing process is utilized to verify the feasibility of the optimized algorithm.

Keywords: NC machining; path optimization; UG; VERICUT.
\end{abstract}

\section{Introduction}

Numerical control machining is an automatic machining technology that uses digital technology and program to control machine, The enhancement includes NC (numerical control), CNC (computer numerical control), FMC (flexible manufacturing cell), FMS (flexible manufacturing system), FA (factory automation), CIM (computer integrated manufacturing), it has become an significant symbol used to measure the level of mechanical manufacturing industry for a country [1]. With the progress of science and technology, complex curved surface is more and more extensive to be used in various fields, such as moulds, tools, energy, transportation, aerospace and Marine, and the development and implementation of the complicated curved surface is more dependent on the complex curved surface's design and manufacturing technology [2]. In the machining of complex curved surface, the tool path is a vital factor that affects the processing quality and efficiency [4].

The paper presents a new generation algorithm for tool path based on optimization of traditional algorithms, thus, ensuring the processing quality, at the same time, improving the processing efficiency.

\section{Algorithms for Tool Path}

Planning technology for tool path is the core content of NC machining. Many scholars both at home and abroad have been studied on it. Now, there are many related algorithms in NC machining, the common algorithms are as follows:

\subsection{Iso-parametric Line Method}

Iso-parametric line method is shown in Figure1. It is the

\footnotetext{
a Corresponding author: Xinghua Niu, niu_x_h@126.com
}

main method of tool path generation in multi-axis $\mathrm{NC}$ machining which is firstly proposed by Loney GC and Ozosy TM [6]. The advantage of this method is that the calculation is simple and fast. However, the defect is that the tool path arrangement is dense, and the machining efficiency is low.

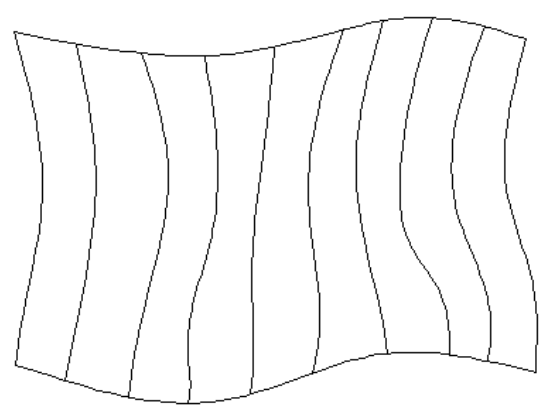

Figure 1. Simulation results.

\subsection{Iso-metric Section Plane Method}

Iso-metric section plane method is shown in Figure 2. It utilizes a set of parallel plane and curved surface intersection, delivered by line as a tool path. The advantage of this approach is that can handle complex curved surface, and the tool path is uniform, this method is commonly used in three-dimensional NC machining. But it is difficult to make the intersect operation between surfaces, and the cross section spacing is difficult to control, leading to dense trajectory in the flat section of surface, sparse trajectory in the steep section. And the machining efficiency is low. 


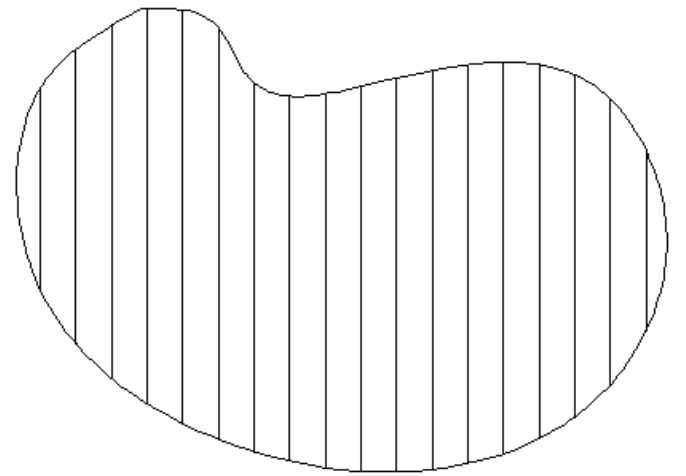

Figure2. Iso-metric section plane method.

\subsection{Iso-scallop Method}

Iso-scallop method is proposed by K.Suresh and D.C.H.Yang in the University of California, 1994. The basic idea is that to ensure the roughness between adjacent lines equal to the allowed maximum of residual height (as shown in Figure 3), so it can shorten the whole path by increasing the step distance of the tool, and save processing time [7]. Obviously, this method has more efficiency than the iso-parametric line method. But this method also has its own defects, such as large calculated amount, the tool path is scattered, and it's not conducive to high speed machining, etc.

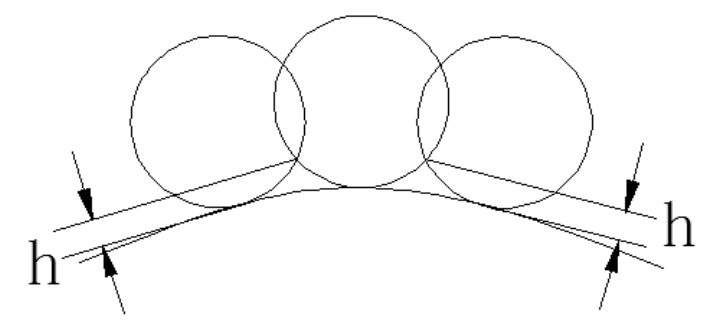

Figure3. Definition of Iso-scallop.

\subsection{Iso-metric Offset Method}

Iso-metric offset method is shown in Figure 4, it is also called boundary offset method. The iso-metric curve of the boundary is as a tool path, the spacing also is determined based on the maximum residual height allowed. The advantage of this method is that the calculation is simple, and the tool path is even and neat, but the machining efficiency is low.

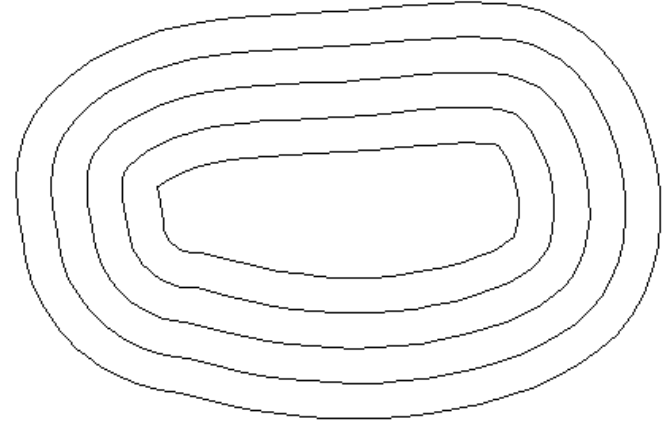

Figure4. Iso-metric offset method.

\section{A New Algorithm for Tool Path}

The traditional generation methods for tool path have their own shortcomings, so there are many studies on improvement of it. For example, the reference [8] presents a generation algorithm for tool path with auto-adaptive iso-parametric line. In order to improve the machining efficiency, this paper presents a new algorithm.

\subsection{Path Spacing Formula}

Path spacing is the distance between two adjacent tool paths, and it is an important factor that influences the accuracy and efficiency of surface machining. If the path spacing is too small, the machining efficiency will be low, on the contrary, it will lead residual height to increase, so how to determinate path spacing is an important problem for surface machining. Reference [1] presents a path spacing formula, as shown in Figure 5:

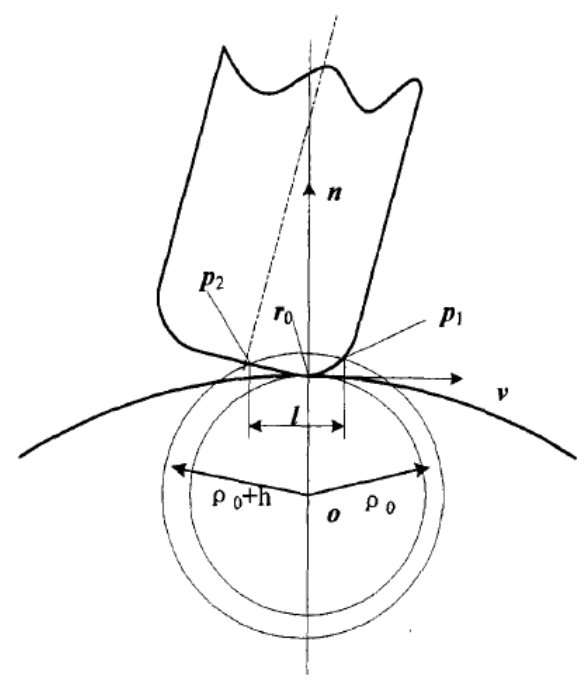

Figure 5. Tool path spacing.

Tool path spacing formula:

$$
|l|=2 \sqrt{2 R h /\left(1-R / K_{0}\right)}
$$

Where $R$ is tool radius, $h$ is the maximum residual 
height, and $k_{0}$ is normal curvature radius.

\subsection{Optimization Algorithm for Tool Path}

Assuming constant of else parameters, it can be seen from the Eq. (1), the greater maximum residual height is given, the larger tool path spacing is got, and obviously, the higher machining efficiency will be also acquired. But for complex curved surface, different area has different feature, as a result, both the curvature radius and the maximum residual height allowed are different. Iso-metric offset method ensures the machining quality, but the machining efficiency is low. Iso-scallop method not only ensures the roughness between adjacent lines equal to the maximum allowable residual height, but also improves the machining efficiency, however, the tool path of this kind of method is scattered, and it's not conducive to high speed machining.

According to the above viewpoints, this paper presents a new generation algorithm for tool path based on iso-metric offset method and iso-scallop method. That is, when the tool path is generated with the iso-scallop method, the given residual height range replaces the allowable maximum of residual height. This method not only retains the iso-scallop method's advantages of high machining efficiency, but also reflects the advantages of the iso-metric offset method. Thus, the tool path is even and neat relatively. So this new algorithm can ensure the processing quality and improve processing efficiency. Concrete flow chart of optimization algorithm for tool path is shown in Figure 6.

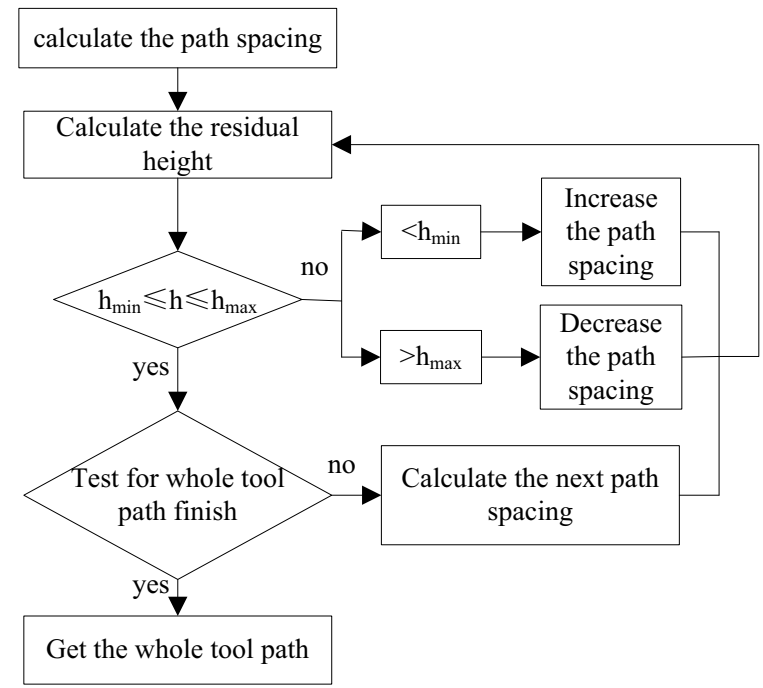

Figure 6. Flow chart of optimization algorithm.

\section{Test for Optimization Effect Based on UG}

In order to verify the optimization effect, an impeller is selected as the processing object in this paper. Then, with the UG software, comparing the total length of the tool path generated respectively by the iso-metric offset method and by the new algorithm, the effect of optimization for the tool path can be shown.
Firstly, using the secondary development platform of UG software, programming the new generation algorithm for tool path (as shown in Figure 7 and Figure 8), and compiling the files of dynamic link library that can be performed by the UG software. Where the maximum of residual height is $0.005 \mathrm{~mm}$, and the minimum is 0.004 $\mathrm{mm}$.

and UF_terminate 0 .

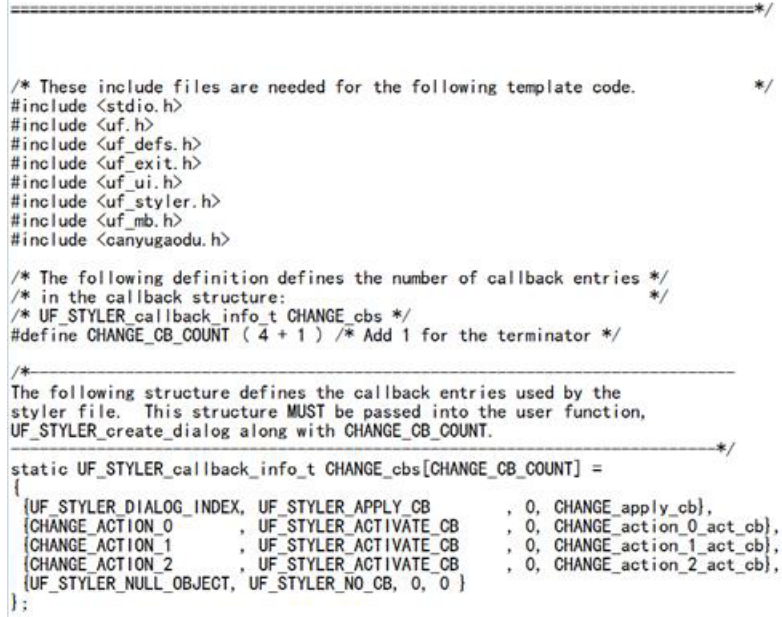

Figure 7. The dialog box code.

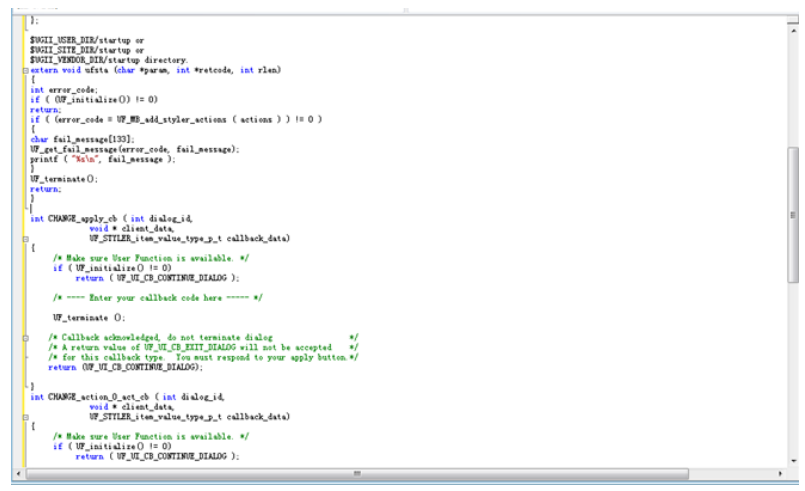

Figure8. Import callback function.

Then by the UG software, following these steps: building three-dimensional modeling, establishing tools, arranging process, and selecting machine tool, etc. Finally, the NC machining program and the tool path is generated automatically. The tool path generated respectively by the iso-metric offset method and by the new algorithm on one blade is shown in Figure 9 and Figure 10.

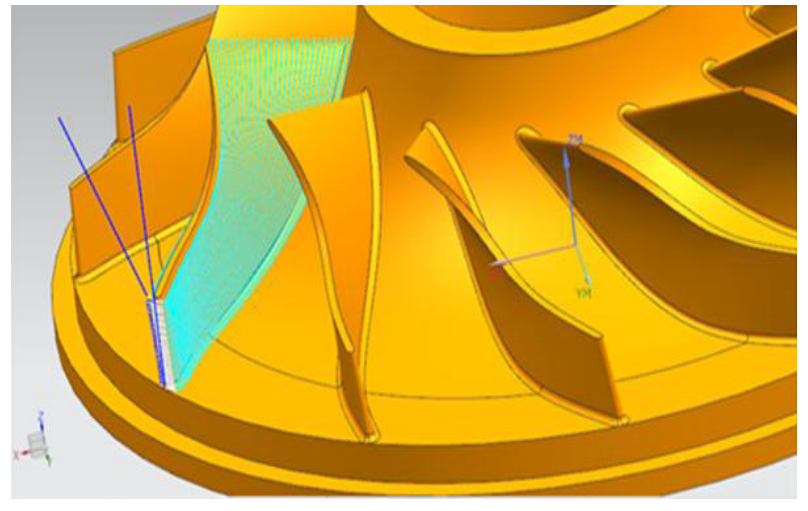


Figure9. Tool path by the iso-metric offset method.

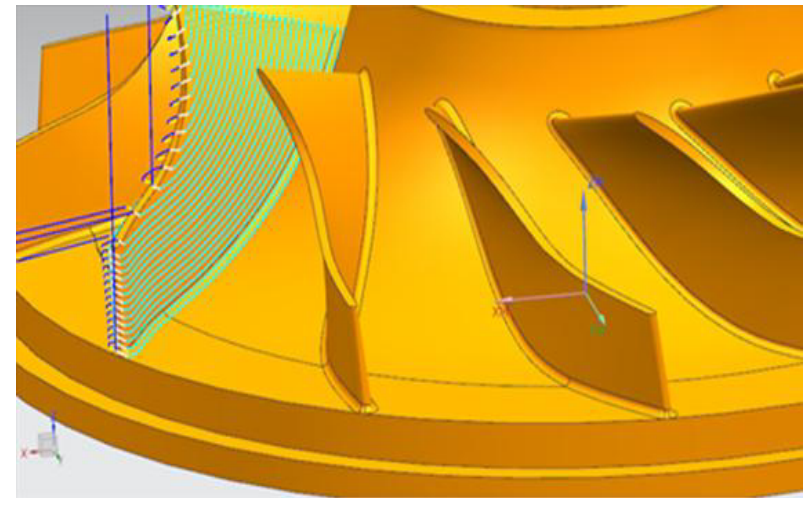

Figure 10. Tool path by the new algorithm.

Comparison result is shown in table 1:

Table 1. Comparison result of tool path.

\begin{tabular}{|c|c|c|}
\hline Algorithm & $\begin{array}{c}\text { Iso-metric offset } \\
\text { method }\end{array}$ & $\begin{array}{c}\text { New } \\
\text { algorithm }\end{array}$ \\
\hline $\begin{array}{c}\text { Total length of } \\
\text { tool path }\end{array}$ & $5680 \mathrm{~mm}$ & $4550 \mathrm{~mm}$ \\
\hline
\end{tabular}

Seen from Figure 9, Figure 10 and table 1, the total length for tool path generated by the new algorithm is shorter than it by the iso-metric offset method, so the new algorithm achieves the purpose for improving machining efficiency.

\section{Machining Simulation Based VERICUT}

VERICUT is 3D software which can perform machining simulation and program optimization. It can avoid the cumbersome process of trying machining, predict the processing results effectively, improve the efficiency of programming, short the manufacturing cycle, and reduce the production cost [10]. In this section, by simulating the machining process with the VERICUT software based on the steps of Sec. 4, it is to test the possible phenomenon of interference or collision, verify the accuracy of $\mathrm{NC}$ program, and monitor the whole simulation process and result.

The model of machine tool for this simulation is HERMLE C42U, that is five-axis linkage NC machine tool, and its control system is HEIDENHAIN. Then, following these steps: importing machine tool, establishing work-blank, building tools' library, and setting processing parameters, etc. Finally, the simulation result is shown in Figure 11. The simulation process denotes the new algorithm is correct and feasible.

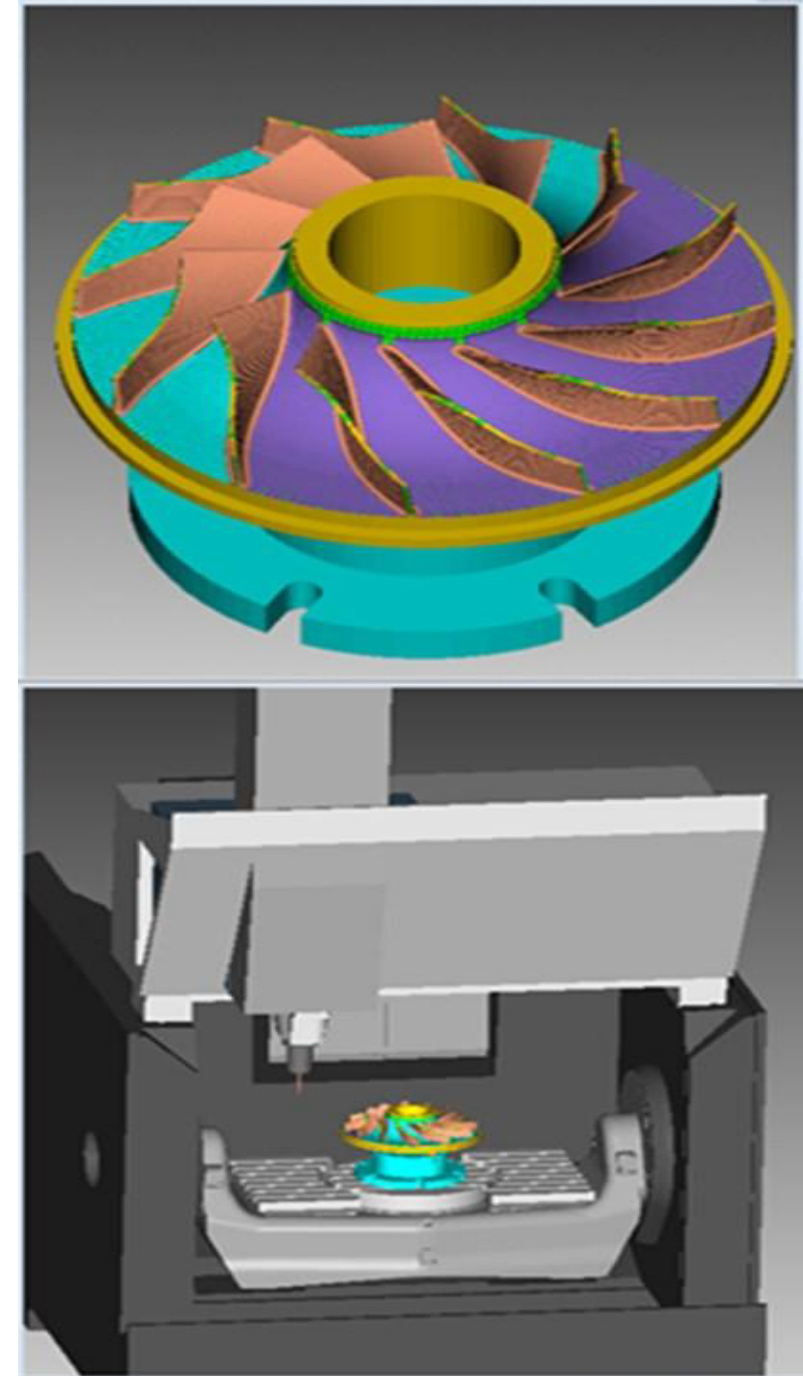

Figure 11. Simulation result.

\section{Conclusions}

A new generation algorithm for tool path was got based on optimization and shortcomings analyses of traditional methods. The 3D software UG is utilized to generate tool path on an impeller, and making contrasts and verifications to the tool path generated by different methods, the result shows that the efficiency of the new algorithm enhanced obviously. The VERICUT software is also used to simulate the whole machining process, and the result verifies the feasibility of the new generation algorithm for tool path.

\section{References}

1. Eungki Lee. Contour offset approach to spiral toolpath generation with constant scallop height. Computer-Aided Design 35, 511,518(2003)

2. Zhou Zhixiong, Zhou Qinyuan, and REN Yinghui. Current Research and Development Trends of Complex Surface Machining Technology. Journal of Mechanical Engineering17, 105,113(2010)

3. Sun Yao. The Preliminary Research on the Optimization of Tool Path and Avoid Overcut. Metal 
Working 21, 42,44(2013)

4. Yue Ying, Han Qingyao, and JIA Jun. Cutter Locus Generation of Sculptural Surface in NC Machining. Machine Tool \&Hydraulics 6, 25,27, 48(2008)

5. Yu Duo. Study on Generation Algorithm for Tool Path in Multi-axis NC Machining. Science and Technology 8, 111(2014)

6. Loney. GC, and Ozsoy. TM. NC Machining of Free form Surfaces. Computer-Aid Design 2,85,90(1987)

7. K. Suresh, and D. C. H. Yang. Constant Scallop-height Machining of Free-form Surfaces.
Journal of Engineering for Industry 5,253,259(1994)

8. Wang Zhixing, and Wang Zhe. Study on a New Algorithm of Tool Path Generation in NC Machining. Journal of Machine Design 11, 20,22(2000)

9. Li Haojie. Process Analysis and Programming for Blade Parts in Multi-axis Machining. Scientific Consult 10, 56,58(2014)

10. Yao Yunping, and Wang Sumei. Research on Modeling Technology of Virtual Machine Tools and Complex Surface Machining Based on VERICUT. New Technology \& New Process, 37,39(2010) 